\title{
Commentaire
}

\section{Réaction rapide face à la COVID-19 : relever les défis et améliorer la préparation mentale du personnel de sécurité publique}

\author{
Alexandra Heber, M.D. (1,2); Valerie Testa, B. Éd. (3,7); Lorraine Smith-MacDonald, Ph. D. (4,5); \\ Suzette Brémault-Phillips, Ph. D. (4,5); R. Nicholas Carleton, Ph. D. $(6,7)$
}

Publié en ligne le 9 septembre 2020

Diffuser cet article sur Twitter

\section{Introduction}

La pandémie de COVID-19 a mis en évidence le rôle essentiel du personnel de sécurité publique (PSP) dans le service et la protection de l'ensemble des Canadiens. Ce personnel est constitué des professionnels qui assurent la sécurité des Canadiens, comme les agents des services frontaliers, les préposés aux communications de la sécurité publique (p. ex. les répartiteurs d'urgence), les travailleurs correctionnels, les pompiers (de carrière et bénévoles), les gestionnaires des urgences autochtones, le personnel opérationnel et $\mathrm{du}$ renseignement, les ambulanciers et les policiers (municipaux, provinciaux et fédéraux $)^{1,2}$. Il travaille souvent aux côtés de fournisseurs de soins de santé ${ }^{1}$ et joue un rôle essentiel dans la gestion de la pandémie de COVID-19. Avant la COVID-19, le PSP faisait déjà état d'expositions répétées à des événements potentiellement traumatisants sur le plan psychologique (EPTP) ${ }^{2}$ ainsi qu'à des facteurs de stress professionnel importants comme le manque de personnel, l'insuffisance des ressources et la fatigue ${ }^{3,4}$. On peut donc raisonnablement s'attendre à ce que les facteurs de stress omniprésents liés à la pandémie de COVID-19, comme le risque de contagion personnelle et familiale (mentionnés dans une conversation du 20 juillet 2020 entre le commandant S. Leduc et Valerie Testa), augmentent encore la pression s'exerçant sur ce personnel.

Chaque secteur dans lequel évolue un PSP (police, services d'incendie, services d'ambulance, services correctionnels, etc.) a besoin d'un soutien adapté pour gérer à long terme les facteurs de stress liés à la pandémie de COVID-19. De plus en plus de publications font état des effets des épidémies précédentes (comme le SRAS ou le SRMO) et de la pandémie actuelle sur les travailleurs de la santé5,6, mais très peu décrivent l'impact de ces pandémies et des épidémies sur le PSP. La collecte de données spécifiques au PSP en vue de générer des éléments de preuve au cœur de la crise de la COVID-19 serait instructive, mais nécessiterait de consacrer du temps et de l'attention à la recherche au détriment de la prestation de services essentiels. Comme il était nécessaire de trouver une autre approche pour synthétiser rapidement les connaissances fondées sur des données probantes et évaluées par des pairs tout en diffusant des stratégies de soutien au PSP, on a mis en place le Projet de soutien et de préparation face à la COVID-19 (PSPC), de l'Institut canadien de recherche et de traitement en sécurité publique (ICRTSP), avec un important soutien financier de la part d'Anciens Combattants Canada (ACC). Il s'agit d'un centre national de connaissances en ligne qui fournit des
Points saillants

- La pandémie de COVID-19 a mis en évidence le rôle essentiel du personnel de sécurité publique dans le service et la protection de l'ensemble des Canadiens.

- Le personnel de sécurité publique signalant déjà des problèmes de santé mentale et de bien-être avant même la pandémie de COVID-19, les nouveaux facteurs de stress pourraient augmenter les besoins en ressources supplémentaires chez ce personnel afin qu'il soit capable de nous aider à long terme.

- Cet article propose divers éléments de soutien pouvant constituer des pistes pertinentes en vue de favoriser le bien-être du personnel de sécurité publique pendant la période de stress prolongé provoqué par la pandémie de COVID-19.

- Les autosoins peuvent s'avérer essentiels au maintien de la santé mentale et du bien-être du personnel de sécurité publique pendant la pandémie de COVID-19.

Mots-clés : personnel de sécurité publique, premiers intervenants, COVID-19, formation, communication de crise, leadership, quarantaine, autosoins 
renseignements fiables sur la santé mentale et le bien-être du PSP à partir de consultations menées avec le personnel de première ligne, les dirigeants et les experts en la matière, afin de recenser les défis auxquels le PSP est confronté. Des réponses à ces défis sont ensuite fournies à partir des publications existantes évaluées par les pairs sur l'impact des pandémies et des épidémies sur les travailleurs de la santé, ainsi qu'à partir des données générales relatives à la santé mentale en cas de catastrophe. Ces résultats sont ensuite adaptés au PSP et diffusés en ligne par les membres du PSP, les cliniciens et les chercheurs sous diverses formes, que ce soit des contenus écrits accessibles, des assemblées virtuelles interactives dirigées par des experts en la matière et des intervenants du PSP, des vidéos ou encore des exercices guidés de gestion du stress en format audio.

\section{Objectif}

Cet article décrit les cinq domaines prioritaires recensés par le PSPC pour soutenir le PSP pendant la COVID-19. L'objectif est de faire connaître le vécu du PSP et d'encourager l'innovation collective en matière de soutien au bien-être de ses membres. Les priorités et les recommandations énumérées ici ne sont pas exhaustives, définitives ou mutuellement exclusives et elles ne sont pas non plus applicables à tous les secteurs ou organismes dans lesquels évoluent les membres du PSP. Il s'agit plutôt de fournir des points de départ à envisager pour continuer à soutenir les membres du PSP, leurs familles et leurs dirigeants. Le tableau 1 présente un résumé de ces recommandations.

\section{Soutenir le PSP pendant la COVID-19}

\section{Formation à la préparation spécialisée}

Le PSP intervient dans des environnements où règne une forte incertitude ${ }^{7}$, ce qui renforce les risques pour la santé mentale liés à des expositions répétées aux EPTP ${ }^{3}$. Les organismes de PSP pourraient être en mesure de soutenir l'efficacité du PSP tout en atténuant les risques liés aux $\mathrm{EPTP}^{3}$ par des formations à la préparation formelle et informelle ${ }^{8,9}$ ainsi que par des formations en santé mentale ${ }^{4}$. Les formations à la préparation par la « répétition » peuvent permettre au PSP de se familiariser avec les EPTP, de réduire l'effet de surprise et d'atténuer la réaction

TABLEAU 1

\section{Récapitulatif des exemples de soutien}

Formation à la préparation spécialisée

Offrir des possibilités d'apprentissage

au moment pertinent

Fournir des aides à la décision

Garder en tête qu'il s'agit d'un marathon

\begin{tabular}{|ll} 
& $\begin{array}{l}\text { Encourager les personnes à prendre soin d'elles-mêmes (p. ex. } \\
\text { exercice, repos, déconnexion du travail, socialisation). }\end{array}$ \\
\hline Communication & $\begin{array}{l}\text { Prévoir une communication fréquente, cohérente, transparente } \\
\text { et interactive. }\end{array}$
\end{tabular}

Maintenir la communication

Prévoir du temps pour les questions, les discussions et les suggestions.

Offrir de l'empathie Souligner l'importance et la valeur de chacun.

Tirer profit des médias

Leadership et travail d'équipe

Rester visible, disponible et bien informé

Combler les besoins fondamentaux

Fournir des messages réguliers qui mettent l'accent sur la compréhension et le désir d'aider.

Utiliser des outils médiatiques spécifiques et cohérents pour la diffusion de l'information.

Combler les besoins fondamentaux

Offrir un soutien social

Maintenir la visibilité de dirigeants de confiance engagés dans une communication empathique, constructive et solidaire.

Trouver des solutions novatrices pour aider les personnes à déterminer comment répondre à leurs besoins fondamentaux malgré les nouvelles complications.

\begin{tabular}{ll} 
Quarantaine & Soutenir les efforts de soutien par les pairs. \\
\hline Garder le contact & $\begin{array}{l}\text { Garder un contact à distance régulier avec le personnel en } \\
\text { quarantaine, en insistant sur le fait qu'il est indispensable et } \\
\text { apprécié. }\end{array}$
\end{tabular}

Penser aux principes fondamentaux Soutenir les membres du PSP auto-isolés en leur garantissant un accès à des services, à l'aide sociale, à des soins médicaux et la fourniture d'alimentation.

Lutter contre la violence conjugale et Être conscient du risque accru de violence conjugale et d'abus la toxicomanie d'alcool et d'autres substances; rappeler aux personnes de demander de l'aide rapidement et leur indiquer où elles peuvent obtenir cette aide.

Prendre soin de soi

Réagir par des choix santé

Mettre l'accent sur les habitudes saines en matière de sommeil, d'alimentation, d'hydratation et d'exercice.

Utiliser des techniques de relaxation et de gestion du stress (p. ex. respiration diaphragmatique et exercices de pleine conscience).

Maintenir ses routines

Maintenir ou établir des routines.

Entretenir des liens sociaux sains et se faire aider

Rester en contact avec la famille, les amis et les collègues.

Faire appel, au besoin, au soutien de membres fiables de la famille, de collègues et de services de soins de santé fondés sur des données probantes.

Abréviation : PSP, personnel de sécurité publique.

automatique au stress qui consiste à « combattre, fuir ou s'immobiliser » lorsque survient un EPTP ${ }^{10,11}$. La nature inattendue de la crise de la COVID-19 est susceptible d'avoir mis en péril les capacités spécifiques de formation à la préparation de nombreux membres du PSP, mais ces derniers travaillent avec succès pour relever 
les défis rencontrés et tireraient profit de soutien supplémentaire dans la poursuite de leurs efforts alors même que la pandémie perdure. Voici diverses suggestions de mesures de soutien additionnelles.

\section{Offrir des possibilités d'apprentissage} au moment pertinent. La formation à la préparation en matière de mesures d'intervention d'urgence pour la COVID-19 a nécessairement été compromise par l'apparition soudaine de cette maladie et le manque de temps disponible pour préparer le personnel. Toutefois, le PSP tirera certainement profit de synthèses des données à jour, de possibilités de formations régulières et brèves ( $p$. ex. une fois par semaine lors d'une réunion quotidienne; à l'aide d'une courte séquence vidéo) proposées par des autorités respectées à propos des dernières lignes directrices, sur les faits nouveaux et sur les stratégies d'intervention les plus récentes ${ }^{12-14}$. Une formation régulière est susceptible de contribuer à réduire l'anxiété, à accroître la confiance accordée à la direction, à renforcer l'image de l'organisme et à diminuer le sentiment d'isolement ${ }^{13,14}$.

2. Fournir des aides à la décision. Des moyens mnémotechniques, des arbres ou des algorithmes de décision officiels, s'ils sont approuvés par les dirigeants et spécifiques aux défis liés à la COVID-19, sont susceptibles de réduire l'incertitude et l'anxiété du PSP dans la crise actuelle ${ }^{4,15}$. Cette information peut être mise à jour au fur et à mesure que de nouvelles données probantes sont disponibles.

3. Garder en tête qu'il s'agit d'un marathon. Se préparer durant la pandémie implique de trouver son rythme face au «marathon » qu'elle nécessite sur le long terme, tout en étant capable d'effectuer des "sprints » intermittents pour mener les interventions d'urgence ${ }^{16}$. Les membres du PSP peuvent avoir besoin d'encouragements supplémentaires pour surveiller leur santé et prendre soin d'eux-mêmes, en faisant de l'exercice, en se reposant, en déconnectant du travail et en socialisant.

\section{Communication}

Faire parvenir la bonne information aux bonnes personnes et au bon moment pouvant s'avérer délicat dans les circonstances les plus favorables, cette tâche risque d'être encore plus difficilement réalisable en situation de pandémie. La " communication de crise » vise à transmettre efficacement l'information dans le chaos qui règne lors d'une situation de crise. Les communications doivent être faciles à comprendre et cohérentes pour que les gens se souviennent des messages clés et prennent des mesures efficaces. Les autorités sanitaires fédérales telles que les Centers for Disease Control and Prevention des États-Unis ${ }^{17}$ fournissent des lignes directrices en soutien aux tentatives de communication de crise efficace. Voici diverses mesures de soutien possibles.

1. Maintenir la communication. Maintenir une communication fréquente, cohérente, transparente et interactive. Une nouvelle information est mieux assimilée et traitée lorsque le contenu est concis, cohérent et fourni par une source respectée ${ }^{18}$. Prévoir du temps pour les questions, les discussions et les suggestions crée un espace qui peut aider les dirigeants à mieux comprendre les besoins du PSP, ce qui est important pour maintenir les capacités de ce dernier pendant un tel marathon ${ }^{7}$. Une communication interactive régulière avec les dirigeants peut aider à renforcer l'estime de soi des membres de l'équipe tout en soulignant que leurs efforts sont reconnus et valorisés.

2. Offrir de l'empathie. La crise liée à la pandémie est susceptible d'engendrer des réactions de peur ou de colère chez les personnes avec lesquelles le PSP interagit. Faire preuve de compréhension empathique et témoigner d'un désir d'aider a le pouvoir de désamorcer ces émotions intenses ${ }^{19}$. Cela étant dit, la pandémie pourrait compliquer les efforts déployés par le PSP pour offrir de l'empathie (p. ex. les masques peuvent interférer avec les indices non verbaux), nécessitant un effort supplémentaire de la part de ses membres, augmentant ainsi la pression qui s'exerce sur eux. Malgré ces efforts supplémentaires requis, surmonter les obstacles liés à la pandémie afin de trouver des moyens de faire preuve d'empathie et de bienveillance peut faciliter l'obtention de résultats positifs pour le PSP comme pour les personnes qu'il sert.
3. Tirer profit des médias. Les médias peuvent contribuer à susciter des comportements altruistes, éthiques et civiques, favorisant ainsi la santé des collectivités en temps de crise ${ }^{18}$. Dans la mesure du possible, il convient de choisir un petit nombre de sources fiables d'information afin de maximiser le nombre de personnes touchées tout en permettant au PSP de savoir aisément où trouver l'information. Les médias traditionnels et sociaux peuvent transmettre des renseignements importants pour optimiser la sécurité publique pendant la pandémie. Les communications avec les médias peuvent également être utilisées pour atténuer les préjugés et les critiques dont peuvent faire l'objet les membres du PSP et d'autres personnels de première ligne lorsqu'ils prennent des décisions urgentes dans un contexte sans précédent, qui nécessite souvent l'élaboration de nouvelles pratiques exemplaires en temps réel ${ }^{18}$.

\section{Leadership et travail d'équipe}

Les dirigeants peuvent jouer un rôle essentiel en soutenant la résilience psychologique et le moral de leurs équipes. Faciliter l'accès rapide à des services de santé mentale fondés sur des données probantes après une atteinte à la santé mentale est important ${ }^{5,20}$. Ne serait-ce que rappeler aux membres du PSP qu'ils peuvent bénéficier d'un soutien avant, pendant et après un EPTP, en particulier un EPTP prolongé comme la pandémie de COVID-19, peut contribuer à atténuer le stress, l'épuisement professionnel et d'autres atteintes à la santé mentale ${ }^{21-23}$. Voici quelques recommandations fondées sur des données probantes à l'intention des dirigeants.

1. Rester visible, disponible et bien informé. La visibilité de dirigeants de confiance peut soutenir le moral et renforcer la conviction d'un soutien disponible, ce qui peut renforcer à son tour la résilience. Les dirigeants qui offrent la preuve, même brièvement, qu'ils connaissent les facteurs de stress touchant l'ensemble de leurs équipes ainsi que les facteurs de stress individuels touchant certains membres des équipes peuvent être très efficaces. Les dirigeants peuvent essayer d'apporter leur aide en rappelant au PSP que la COVID-19 est un 
EPTP prolongé qui nécessite des objectifs réalisables à court et à long terme, ainsi qu'une concentration sur les activités pérennes. Les dirigeants peuvent aider les membres du personnel à trouver un sens à la situation, à se concentrer au-delà de la crise actuelle et à être fiers de savoir qu'ils contribuent au bien de tous dans leur collectivité et leur pays ${ }^{16,24}$.

2. Combler les besoins fondamentaux. Les besoins fondamentaux des membres du PSP doivent toujours être comblés afin qu'ils puissent accomplir leurs tâches efficacement, mais la pandémie a entraîné des complications, comme l'auto-isolement, susceptibles de compromettre leurs capacités à combler des besoins fondamentaux comme la garde d'enfants ou les courses à l'épicerie. Les dirigeants et les organismes peuvent apporter leur aide en s'efforçant de recenser ces difficultés pour proposer des solutions novatrices qui compenseront ces nouvelles complications ${ }^{25}$.

3. Offrir un soutien social. Le soutien social peut s'avérer important pour la résilience, mais certaines dimensions de ce soutien social peuvent être altérées par la nécessité de respecter les règles de distanciation et l'autoisolement ${ }^{25,26}$. Les dirigeants peuvent contribuer à renforcer les relations positives et le moral des membres du PSP en soulignant le soutien que ces derniers apportent à leurs équipes, en encourageant le soutien entre collègues au sein des équipes et, si possible, en mettant en place des équipes et des cycles de travail stables et cohérents $^{25-27}$.

\section{Quarantaine}

Les données probantes tirées des précédentes épidémies associent l'isolement à une santé mentale fragilisée $e^{6,18,28,29}$. La quarantaine ou l'auto-isolement peuvent conduire à la solitude, l'ennui, la peur, la perte de routine et avoir diverses conséquences comme la consommation excessive d'alcool et d'autres substances ${ }^{30-32}$. Plusieurs cycles d'isolement obligatoire pendant une pandémie peuvent également exacerber des problèmes de santé mentale préexistants ${ }^{5}$. Après l'isolement, le retour au travail peut être source d'anxiété en raison des craintes de (ré) infection et des réactions des collègues, qui ont pu se trouver dans l'obligation de faire des heures supplémentaires pour pallier le manque de personnel. Voici certaines options susceptibles de réduire les effets néfastes de l'isolement.

1. Garder le contact. Le personnel d'un lieu de travail peut soutenir l'un de ses membres isolé en restant en contact et en prenant régulièrement de ses nouvelles à distance. Affirmer que les personnes isolées demeurent des membres à part entière et appréciés de l'équipe peut contribuer à atténuer les problèmes potentiels de santé mentale.

2. Penser aux principes fondamentaux. Parmi les mesures qui soutiennent le PSP pendant un auto-isolement, citons le fait de faciliter l'accès aux services et aux fournitures à distance (aide sociale, soins médicaux, commande de courses d'épicerie, etc.).

3. Lutter contre la violence conjugale et la toxicomanie. La pandémie de COVID-19 a entraîné une augmentation des cas de violence conjugale et des cas d'abus d'alcool et d'autres substances ${ }^{30-32}$. On doit informer les membres du PSP de ce risque accru, leur rappeler qu'ils doivent demander de l'aide rapidement et leur permettre un accès à des ressources adaptées.

\section{Prendre soin de soi}

Il peut être particulièrement difficile de prendre soin de soi lorsque l'on est confronté à des facteurs de stress professionnels comme le travail par quarts, les horaires irréguliers et l'épuisement. Le stress supplémentaire lié à la COVID-19 peut toutefois être partiellement atténué en invitant régulièrement les membres du PSP à s'engager dans des pratiques quotidiennes d'autosoins pour soutenir leur bien-être, en particulier leur santé mentale et leur résilience ${ }^{33,34}$. Voici quelques suggestions pour prendre soin de soi.

1. Réagir par des choix santé. Rappelez aux membres du PSP qu'ils doivent s'efforcer de bien dormir, de bien manger et de se reposer régulièrement. Utiliser des techniques de relaxation et de gestion du stress (comme la respiration diaphragmatique et les exercices de pleine conscience) favorise le maintien d'un sentiment de bien-être. Ne serait-ce que 30 secondes de respiration profonde ou 5 minutes d'exercice de pleine conscience pendant les pauses et avant le sommeil peuvent s'avérer utiles.

2. Maintenir ses routines. Le maintien de routines même simples, comme un rituel avant d'aller se coucher ou un entraînement physique programmé régulièrement, peut asseoir un ressenti de structure et de prévisibilité pendant la période d'incertitude prolongée provoquée par la pandémie.

3. Entretenir des liens sociaux sains et se faire aider. Rester en contact avec sa famille, ses amis et ses collègues peut aider à préserver son sentiment d'appartenance collective, sa résilience et son bien-être. Dans la mesure du possible, il convient de donner la priorité aux activités et aux plateformes de médias sociaux en ligne positives, en réduisant le plus possible les activités et les plateformes perturbantes, angoissantes et frustrantes $^{35}$. Faire appel au soutien de membres fiables de la famille, de collègues ou de services de soins de santé fondés sur des données probantes en cas de besoin peut aider les membres du PSP à gérer le stress prolongé causé par la pandémie.

\section{Conclusion}

La pandémie de COVID-19 a mis en évidence notre dépendance à l'égard du nombre relativement restreint de personnes qui travaillent pour maintenir la santé, le bien-être et la sécurité de la population. Elle révèle aussi les difficultés à offrir un soutien aux membres du PSP alors même qu'ils assument un rôle essentiel. La nature, l'ampleur et la portée sans précédent de la pandémie ont exigé la mise en place de solutions appelées à durer, innovantes et constamment à réévaluer. Cet article présente des recommandations en vue de soutenir le PSP qui sont fondées sur des données cliniques et, le cas échéant, sur des données probantes. Ces recommandations sont organisées et diffusées par le biais d'une initiative nationale canadienne, le Projet de soutien et de préparation face à la COVID-19. À mesure de l'évolution de la pandémie de COVID-19 de l'émergence de nouveaux défis, il faudra fournir des efforts concertés pour fournir et maintenir un soutien psychosocial spécifique au PSP, afin de lui permettre non seulement de faire face aux 
nombreux défis à venir, mais aussi de s'épanouir face à ces derniers.

\section{Remerciements}

Les auteurs remercient sincèrement les personnes suivantes pour leurs précieuses contributions : $\mathrm{D}^{\mathrm{re}}$ Cyd Courchesne, Direction générale des professionnels de la santé, Anciens Combattants Canada; Ron Martin, Faculté d'éducation, Université de Regina; Rosemary Ricciardelli, Faculté de sociologie, Université Memorial de Terre-Neuve; Lorraine Downey, Service paramédic d'Ottawa, Ville d'Ottawa/Ottawa First Responder Mental Health Network Collaboration; Christine Godin, Service paramédic d'Ottawa, Ville d'Ottawa; Heather Hadjistavropoulos, Département de psychologie, Université de Regina; $D^{\text {re }}$ Ruth Lanius, Unité de recherche sur le trouble de stress post-traumatique (TSPT), Université Western/Département de psychiatrie, Université Western/École des sciences de la réadaptation, Université McMaster; Margaret McKinnon, Département de psychiatrie et de neurosciences du comportement, Université McMaster/Santé mentale et dépendances, St. Joseph's Healthcare Hamilton/Homewood Research Institute; capitaine Alain Pellegroms, Service des incendies d'Ottawa, Ville d'Ottawa; James M. Thompson, Département des sciences de la santé publique, Université Queen's/ Institut canadien de recherche sur la santé des militaires et des vétérans; les membres du comité du PSPC et les examinateurs du personnel de la sécurité publique. Veuillez consulter le site Internet du PSPC pour obtenir la liste complète des nombreuses personnes que nous tenons à remercier pour leur contribution au Projet de soutien et de préparation face à la COVID-19.

\section{Conflits d'intérêts}

Les auteurs déclarent qu'ils n'ont aucun conflit d'intérêts à signaler.

\section{Contributions des auteurs et avis}

AH, VT, LSM, SBP et NC ont tous contribué à la conception, à la rédaction et à la revue critique de ce commentaire.

Le contenu de l'article et les points de vue qui y sont exprimés n'engagent que les auteurs et ne correspondent pas nécessairement à ceux du gouvernement du Canada.

\section{Références}

1. Gouvernement du Canada. Cadre fédéral relatif au stress post-traumatique. Ottawa (Ont) : Gouvernement du Canada; 2020. [n ${ }^{\circ}$ HP10-34/2020EPDF]. En ligne à : https://www .canada.ca/content/dam/phac-aspc /documents/services/publications /healthy-living/federal-framework -post-traumatic-stress-disorder/pub1 -fra.pdf

2. Institut canadien de traitement et de recherche en sécurité publique (ICRTSP). Glossaire des termes : une compréhension commune des termes courants pour décrire les traumatismes psychologiques (version 2.1). Regina (SK) : ICRTSP; 2019. En ligne à : https://www.cipsrt-icrtsp.ca/fr /wp-content/uploads/sites/6/2020 /02/Glossaire-des-termes-version-2.1 .pdf

3. Carleton RN, Afifi TO, Taillieu, T, et al. Exposures to potentially traumatic events among public safety personnel in Canada. Can J Behav Sci. 2019; 51:37-52. doi:10.1037/cbs0000115.

4. Carleton RN, Afifi TO, Turner S, et al. Mental health training, attitudes toward support, and screening positive for mental disorders. Cogn Behav Ther. 2020;49(1):55-72. doi:10.1080 /16506073.2019.1575900.

5. Walton M, Murray E, Christian MD. Mental health care for medical staff and affiliated healthcare workers during the COVID-19 pandemic. Eur Heart J Acute Cardiovasc Care. 2020; 9(3):241-247. doi:10.1177/204887262 0922795 .

6. Hawryluck L, Gold WL, Robinson S, Pogorski S, Galea S, Styra R. SARS control and psychological effects of quarantine, Toronto, Canada. Emerg Infect Dis. 2004;10(7):1206-1212. doi: 10.3201/eid1007.030703.

7. Camp RD, Mellow R. PSP Leadership and mental health. Presentation at CRRP Virtual Town Hall Series; 2020; Canadian Institute for Public Safety Research and Treatment (CIPSRT). En ligne à : https://www.youtube.com /watch?v = 97-mlI-ugE0
8. Sécurité publique Canada. Protection civile [Internet]. Ottawa (Ont.) : Gouvernement du Canada; 2019 [consultation le 29 mai 2020]. En ligne à : https://www.securitepublique.gc.ca /cnt/mrgnc-mngmnt/mrgnc-prprdnss /index-fr.aspx

9. Perry RW, Lindell MK. Preparedness for emergency response: guidelines for the emergency planning process. Disasters. 2003;27(4):336-350. doi: 10.1111/j.0361-3666.2003.00237.x.

10. Carleton RN. Into the unknown: a review and synthesis of contemporary models involving uncertainty. J Anxiety Disord. 2016;39:30-43. doi: 10.1016/j.janxdis.2016.02.007.

11. Carleton RN, Koroll S, Wagner J, et al. A prospective assessment of PTSD symptoms using analogue trauma training with nursing students. Can J Behav Sci. 2019;51(3):181-191. doi: $10.1037 /$ cbs0000127.

12. Becker AL. World Health Assembly addresses pandemic flu [Internet]. Minneapolis (MN): Center for Infectious Disease Research and Policy; 2005 [mis en ligne le 16 mai 2005, consultation le 18 mai 2020]. En ligne à : https://www.cidrap.umn.edu/news -perspective/2005/05/world-health -assembly-addresses-pandemic-flu

13. O’Donnel R. When it comes to training, timing is everything. HR Dive [Internet]. 2017 [mise en ligne le 17 octobre 2005, consultation le 18 mai 2020]. En ligne à : https:// www.hrdive.com/news / when-it -comes-to-training-timing-is-everything /506971/

14. Mason R, Pegler C, Weller M. A learning object success story. J Asynchron Learn Netw. 2005;9(1):97-105.

15. Rosenbaum L. Facing Covid-19 in Italy - ethics, logistics, and therapeutics on the epidemic's front line. $\mathrm{N}$ Engl J Med. 2020;382:1873-1875. doi: 10.24059/olj.v9i1.1805.

16. Wu AW, Connors C, Everly GS Jr. COVID-19: peer support and crisis communications strategies to promote institutional resilience. Ann Intern Med. 2020;172(12):822-823. doi: 10.7326 /M20-1236. 
17. U.S. Department of Health and Human Services. Crisis emergency and risk communication. Centers for Disease Control and Prevention; 2014. En ligne à : https://emergency.cdc.gov/cerc/ppt /cerc_2014edition_Copy.pdf

18. Lunn PD, Belton CA, Lavin C, McGowan FP, Timmons S, Robertson, DA. Using behavioral science to help fight the coronavirus. JBPA. 2020;3(1):1-15. doi: 10.30636/jbpa.31.147.

19. Decety J, Bartall IB-A, Uzefovsky F, Knafo-Noam A. Empathy as a driver of prosocial behaviour: highly conserved neurobehavioural mechanisms across species. Phil Trans R Soc Lond B Biol Sci [Internet]. 2016 [consultation le 29 mai 2020];371(1686):20150077. doi:10.1098/rstb.2015.0077.

20. Kinman G, Teoh K. What could make a difference to the mental health of UK doctors? A review of the research evidence. London (UK): Society of Occupational Medicine; 2018. En ligne à : https://www.som.org.uk/sites/som .org.uk/files/What_could_make_a_ difference_to_the_mental_health_of_ UK_doctors_LTF_SOM.pdf

21. Maunder RG, Lancee WJ, Balderson KE, Bennett JP, Borgundvaag B, Evans $\mathrm{S}$, et al. Long-term psychological and occupational effects of providing hospital healthcare during SARS outbreak. Emerg Infect Dis. 2006;12(12): 1924-1932. doi:10.3201/eid1212.060584.

22. Brooks SK, Dunn R, Amlôt R, Rubin GJ, Greenberg N. A systematic, thematic review of social and occupational factors associated with psychological outcomes in healthcare employees during an infectious disease outbreak. J Occup Environ Med. 2018;60(3):248-257. doi: 10.1097/JOM.0000000000001235.

23. Yassi A, Moore D, Fitzgerald JM, Bigelow P, Hon C, Bryce E. Research gaps in protecting healthcare workers from SARS and other respiratory pathogens: an interdisciplinary, multistakeholder, evidence-based approach. J Occup Environ Med. 2005;47(1):4150. doi:10.1097/01.jom.0000150207 .18085 .41 .

24. Shanafelt T, Ripp J, Trockel M. Understanding and addressing sources of anxiety among health care professionals during the COVID-19 pandemic. JAMA. 2020;323(21):2133-2134. doi: 10.1001/jama.2020.5893.
25. Naushad VA, Bierens JJ, Nishan KP, et al. A systematic review of the impact of disaster on the mental health of medical responders. Prehosp Disaster Med. 2019;34(6):632-643. doi:10.1017/S1049023X19004874.

26. Brewin CR, Andrews B, Valentine JD. Meta-analysis of risk factors for posttraumatic stress disorder in traumaexposed adults. J Consult Clin Psychol. 2000;68(5):748-766. doi:10.1037// 0022-006x.68.5.748.

27. Ho C, Chee C, Ho R. Mental health strategies to combat the psychological impact of COVID-19 beyond paranoia and panic. Ann Acad Med Singapore. 2020;49(3):155-160.

28. Brooks SK, Webster RK, Smith LE, et al. The psychological impact of quarantine and how to reduce it: rapid review of the evidence. Lancet. 2020; 395:912-920. doi:10.1016/S0140-6736 (20)30460-8.

29. Maunder RG, Leszcz M, Savage D, et al. Applying the lessons of SARS to pandemic influenza. Can J Public Heal. 2008;99(6):486-488. doi:10.1007 /BF03403782.

30. Bradbury-Jones C, Isham L. The pandemic paradox: the consequences of COVID-19 on domestic violence. J Clin Nurs. 2020;29(13-14):2047-2049. doi:10.1111/JOCN.15296.

31. Douglas M, Katikireddi SV, Taulbut M, McKee M, McCartney G. Mitigating the wider health effects of covid-19 pandemic response. BMJ [Internet]. 2020 [consultation le 29 mai 2020];369:m1557. doi:10.1136/bmj .m1557.

32. Humphreys KL, Myint MT, Zeanah $\mathrm{CH}$. Increased risk for family violence during the COVID-19 pandemic. Pediatrics [Internet]. 2020 [consultation le 26 mai 2020];e20200982. doi: 10.1542/peds.2020-0982.

33. Unadkat S, Farquhar M. Doctors' wellbeing: self-care during the covid19 pandemic. BMJ [Internet]. 2020 [consultation le 29 mai 2020];368(1150). doi:10.1136/bmj.m1150.

34. Adams JG, Walls RM. Supporting the health care workforce during the COVID-19 global epidemic. JAMA. 2020; 323(15):1439-1440. doi:10.1001 /jama.2020.3972.
35. Bansal P, Bingermann TA, Greenhawt $\mathrm{M}$, et al. Clinician wellness during the COVID-19 pandemic: extraordinary times and unusual challenges for the allergist/immunologist. J Allergy Clin Immunol Pract. 2020;8(6):1781-1790. e3. doi:10.1016/j.jaip.2020.04.001. 